\title{
Análisis de costo-efectividad en Colombia de anastrazol Vs. tamoxifeno como terapia inicial en mujeres con cáncer temprano de mama y receptor hormonal positivo
}

\author{
Óscar Gamboa1, Sandra Díaz², Liliana Chicaíza³, Mario García³ \\ 1 Subdirección de Investigaciones, Instituto Nacional de Cancerología, Bogotá, D.C., Colombia \\ 2 Grupo de Cirugía de Seno y Tejidos Blandos, Instituto Nacional de Cancerología, Bogotá, D.C., Colombia \\ 3 Facultad de Ciencias Económicas, Universidad Nacional de Colombia, Bogotá, D.C., Colombia
}

Introducción. En países de ingresos altos, el tamoxifeno ha venido siendo reemplazado por los inhibidores de la aromatasa -como el anastrazol- en la terapia hormonal adyuvante en el cáncer temprano de mama con receptor hormonal positivo, por sus mejores resultados en tiempo libre de enfermedad. Es necesario identificar si este cambio resulta costo-efectivo en los países de ingreso medio, como Colombia.

Objetivo. Evaluar el costo-efectividad para Colombia del anastrazol comparado con el tamoxifeno, como terapia inicial por cinco años en mujeres posmenopáusicas con cáncer temprano de mama y receptor hormonal positivo.

Materiales y métodos. Éste es un análisis basado en la literatura. Se utilizó el modelo de Markov para describir la historia natural de la enfermedad y modelar los tratamientos. La efectividad se midió en tiempo libre de enfermedad. Las probabilidades de transición y los efectos secundarios de los fármacos se extrajeron de la literatura. Se tomó la mediana de costos de algunas empresas promotoras de salud y del Instituto Nacional de Cancerología, en pesos colombianos del 2007. Se hizo análisis de sensibilidad probabilística y de una sola vía de las variables de costo.

Resultados. En comparación con el tamoxifeno, la terapia con anastrazol genera un tiempo adicional libre de recaída de 0,49 años; cada año libre de recaída cuesta Col\$27’210.604 y Col\$37’071.337 con una tasa de descuento del $3 \%$. En el caso de efectos mantenidos, las razones de costo-efectividad con descuento y sin él son Col\$23'617.400 y Col\$16'140.282.

Conclusiones. La utilización de anastrazol genera un costo por año libre de recaída superior al producto interno bruto per cápita de Colombia (Col\$ 7’521.363 para 2007). En consecuencia, resulta recomendable para Colombia continuar con el tamoxifeno por cinco años.

Palabras clave: neoplasias de la mama, agentes antineoplásicos hormonales, análisis costo-beneficio, inhibidores de la aromatasa, economía farmacéutica, Colombia

Cost-benefit analysis of anastrazol and tamoxifen in adjuvant treatment of hormone receptorpositive, post-menopausal breast cancer

Introduction. In high-income countries, tamoxifen has been replaced by aromatase inhibitors such as anastrozole in adjuvant hormone therapy for early breast cancer. These drugs target patients with positive hormone receptors, due to the better results achieved for disease-free survival. The costeffectiveness of this treatment change has not been evaluated in middle income countries.

Objective. The cost effectiveness of anastrozole vs tamoxifen was assessed during five-years of adjuvant treatment of hormone receptor-positive, post-menopausal early breast cancer patients.

Materials and methods. This is a literature-based analysis. The natural history of the breast cancer and the effects of treatment were modeled as a Markov process. Effectiveness was defined as disease-free survival. Transition probabilities for the disease and adverse effects were obtained from the literature. Costs were defined as the median of actual costs provided by health insurance companies and the Colombian National Cancer Institute expressed in 2007 Colombian pesos. Probabilistic sensitivity analysis was performed, along with one way sensitivity analysis was for the costs.

Results. Compared with tamoxifen, anastrazol results in an additional relapse-free period of 0.5 years. Each relapse-free year obtained by tamoxifen cost 27,210,604 pesos, or with anastrazol 37,071,337 pesos with a discount rate of $3 \%$. The cost for a sustained-effects scenario were $23,617,400$ pesos for tamoxifen and $\$ 16,140.282$ for anastrazol.

Conclusions. The use of anastrazol has an additional cost per relapse-free year of 7,521,363 pesos (2007). Therefore, for postmenopausal, early breast cancer hormone receptor positive women in Colombia, the cost-effective alternative is tamoxifen used as adjuvant therapy for five years. 
Key words: breast neoplasms; antineoplastic agents, hormonl; cost-benefit analysis, aromatase inhibitors; economics, pharmaceutical; Colombia.

El tamoxifeno ha sido el tratamiento estándar en Colombia de hormonoterapia adyuvante para pacientes con cáncer de mama. Este fármaco ha demostrado disminuir la recurrencia y la mortalidad en mujeres con cáncer de seno con receptor hormonal positivo (1). En la última década se han incorporado a la hormonoterapia otra clase de medicamentos, como los inhibidores de la aromatasa, los cuales, comparados con el tamoxifeno, han demostrado mejoría en la supervivencia libre de enfermedad $(2,3)$.

Ambas opciones presentan efectos secundarios: en el caso del tamoxifeno, se presenta incremento en el riesgo de cáncer de endometrio y tromboembolia; en el caso de los inhibidores, se presenta una mayor frecuencia en el riesgo de osteoporosis, fracturas y eventos cardiovasculares $(4,5)$. Los inhibidores, comparados con el tamoxifeno, no han demostrado beneficios en la terapia inicial en la supervivencia global a cinco años $(6,7)$.

El tratamiento con anastrazol, aunque no se encuentra incorporado dentro del plan de beneficios del sistema de salud de Colombia, se ha prescrito de manera no generalizada sin que haya evaluaciones económicas que sustenten su uso.

Aunque las evaluaciones de costo-efectividad realizadas en países de ingresos altos, como Canadá, Estados Unidos, Reino Unido y Bélgica (8-13), señalan que el pasar de tamoxifeno a anastrazol resulta costo-efectivo. Este artículo busca evaluar si el cambio también resulta costo-efectivo para un país de ingresos medios, como Colombia.

\section{Materiales y métodos}

Se realizó un estudio de costo-efectividad desde la perspectiva del sistema de salud colombiano, comparando el tamoxifeno con el anastrazol como terapia inicial en mujeres posmenopáusicas con cáncer temprano de mama y receptor hormonal positivo.

Para evaluar el nivel de costo-efectividad de la terapia hormonal adyuvante por cinco años

\footnotetext{
Correspondencia:

Liliana Alejandra Chicaíza, Facultad de Ciencias Económicas, Universidad Nacional de Colombia, Carrera 30 № 45-03, edificio 311, oficina 401B Bogotá, D.C., Colombia

Telefax: (571) 316 5000, extensión 12371

lachicaizab@unal.edu.co

Recibido: 03/02/09; aceptado:06/08/09
}

con anastrazol Vs. tamoxifeno, se construyó el modelo de Markov de la historia natural del cáncer temprano de mama para una cohorte hipotética de 1.000 mujeres de 50 años, seguida a lo largo de 30 años. Dado que el objetivo de la terapia adyuvante es disminuir el riesgo de recaída a largo plazo, se estableció este horizonte temporal de 30 años, el cual permite incluir los costos y resultados relevantes. El cáncer de mama es una enfermedad crónica en la que el riesgo futuro de muerte o recaída depende del estado actual de la enfermedad y no de los estados anteriores. Por esta razón, se escogió el modelo de Markov, en el que los cambios sólo dependen del estado actual. En el modelo, las mujeres pueden estar en uno de los nueve estados que aparecen en la figura 1.

Como medida de efectividad se usó la supervivencia libre de enfermedad, definida como el tiempo que transcurre desde el inicio del tratamiento hasta la primera ocurrencia de una recaída local, a distancia, de cáncer del lado contrario o muerte por alguna causa.

Siguiendo la recomendación de algunos autores $(14,15)$, el modelo incluye las probabilidades de recaída local, regional, a distancia, cáncer del lado contrario y las probabilidades de efectos adversos asociadas al tamoxifeno (cáncer de endometrio y tromboembolia) y al anastrazol (fracturas y eventos cardiovasculares).

Las probabilidades de recaída (local-regional, a distancia o cáncer del lado contrario) de los primeros cinco años para el tamoxifeno, se estimaron a partir del estudio ATAC (arimidex, tamoxifen alone or in combination) $(16,17)$, y las probabilidades para el anastrazol se calcularon multiplicando las estimadas para el tamoxifeno por las razones de riesgo (hazard ratio) allí reportadas. Las probabilidades de recaída después del quinto año fueron estimadas del grupo placebo del estudio MA 17 (18).

Las probabilidades de efectos secundarios del tamoxifeno y de los inhibidores de la aromatasa fueron estimadas de los estudios publicados de hormonoterapia adyuvante en cáncer de mama (16-21).

Las probabilidades de muerte por los efectos adversos se estimaron de estudios observacionales y revisiones de la literatura publicadas (22-33). Las probabilidades de morir por otras causas 


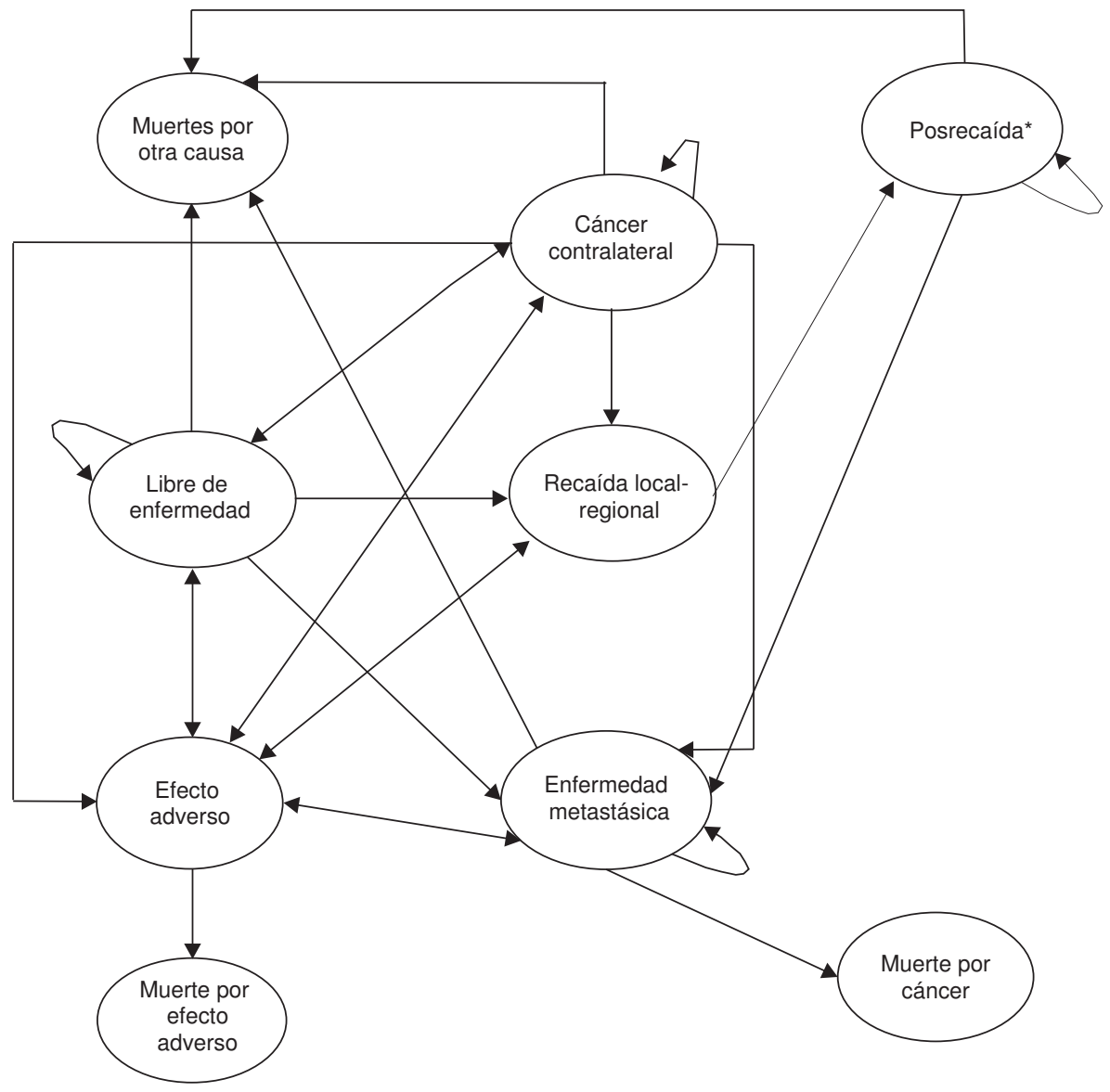

Figura 1. Historia natural de la enfermedad a la primera recaída *Las probabilidades de recaída a distancia dependen del tipo de recaída inicial (local-regional o del lado contrario). Las mujeres con recaída local-regional tuvieron mayor probabilidad de recaída a distancia que las mujeres con cáncer del lado contrario.

se extrajeron de la información reportada por el Departamento Administrativo Nacional de Estadística para el año 2005 sobre estadísticas vitales.

Las probabilidades de transición entre los distintos estados fueron ajustadas anualmente, siguiendo las recomendaciones de Miller (34) (cuadro 1).

El modelo se validó comparando las funciones de supervivencia libres de enfermedad reportadas en el estudio ATAC con las estimadas por el modelo para tamoxifeno. La figura 2 muestra la similitud de las funciones de supervivencia reportadas por el estudio y las reportadas por el modelo.

Bajo la perspectiva del sistema de salud colombiano, se utilizaron costos de mercado para valorar el tratamiento de la enfermedad o cualquier tratamiento para los efectos adversos. La efectividad se midió en tiempo libre de enfermedad y las unidades de costo fueron unidades monetarias (pesos colombianos del 2007). Los costos utilizados en el modelo se presentan en el cuadro 2. Los costos de recaída se tomaron a partir de datos individuales de 23 mujeres con cáncer de mama que presentaron recaída y fueron manejadas en el Instituto Nacional de Cancerología. Para los costos del tratamiento de los efectos adversos, se partió de la mediana de costos de facturación de eventos adversos de diversas empresas prestadoras de salud para un periodo de un año. El rango de costos fue del valor mínimo al máximo observado.

Para evaluar el nivel de costo-efectividad de las estrategias de manejo con hormonoterapia, se calcularon razones de costo-efectividad incremental. Se consideró, siguiendo la recomendación de la Organización Mundial de la Salud, que una estrategia es costo-efectiva si las razones de costo-efectividad incremental son menores que el producto interno bruto per cápita (35), que para Colombia en el 2007 fue de Col\$ 7’521.363 (36). El 
Cuadro 1. Probabilidades estimadas usadas en el modelo.

\begin{tabular}{|c|c|c|c|c|}
\hline \multirow{2}{*}{ Probabilidad anual de recaída del lado contrario con tamoxifeno } & \multirow[t]{2}{*}{$\begin{array}{l}\text { Escenario } \\
\text { base }\end{array}$} & \multicolumn{2}{|c|}{$\begin{array}{l}\text { Rango de análisis } \\
\text { de sensibilidad }\end{array}$} & \multirow[t]{2}{*}{ Fuente } \\
\hline & & & & \\
\hline 1 a 3 años & 0,0043 & 0,0020 & 0,0066 & $16-18$ \\
\hline 3 a 5 años & 0,0023 & 0,0006 & 0,0040 & $16-18$ \\
\hline Más de 5 años & 0,0043 & 0,0016 & 0,0071 & $16-18$ \\
\hline \multicolumn{5}{|l|}{ Probabilidad anual de recaída local-regional con tamoxifeno } \\
\hline 1 a 3 años & 0,0109 & 0,0072 & 0,0146 & $16-18$ \\
\hline 3 a 5 años & 0,0046 & 0,0022 & 0,0070 & $16-18$ \\
\hline Más de 5 años & 0,0051 & 0,0022 & 0,0081 & $16-18$ \\
\hline \multicolumn{5}{|l|}{ Probabilidad anual de recaída a distancia con tamoxifeno } \\
\hline 1 a 3 años & 0,0243 & 0,0188 & 0,0298 & $16-18$ \\
\hline 3 a 5 años & 0,0187 & 0,0139 & 0,0236 & $16-18$ \\
\hline Más de 5 años & 0,0147 & 0,0097 & 0,0197 & $16-18$ \\
\hline \multicolumn{5}{|l|}{ Probabilidad anual de recaída del lado contrario con anastrazol } \\
\hline 1 a 3 años & 0,0036 & 0,0014 & 0,0064 & $16-18$ \\
\hline 3 a 5 años & 0,0020 & 0,0004 & 0,0040 & $16-18$ \\
\hline Más de 5 años & 0,0043 & 0,0016 & 0,0071 & $16-18$ \\
\hline Escenario efectos mantenidos & 0,0037 & 0,0012 & 0,0070 & $16-18$ \\
\hline \multicolumn{5}{|l|}{ Probabilidad anual de recaída local-regional con anastrazol } \\
\hline 1 a 3 años & 0,0091 & 0,0051 & 0,0141 & $16-18$ \\
\hline 3 a 5 años & 0,0039 & 0,0017 & 0,0069 & $16-18$ \\
\hline Más de 5 años & 0,0051 & 0,0022 & 0,0081 & $16-18$ \\
\hline Escenario efectos mantenidos & 0,0044 & 0,0016 & 0,0080 & $16-18$ \\
\hline \multicolumn{5}{|l|}{ Probabilidad anual de recaída a distancia con anastrazol } \\
\hline 1 a 3 años & 0,0202 & 0,0134 & 0,0287 & $16-18$ \\
\hline 3 a 5 años & 0,0161 & 0,0105 & 0,0233 & $16-18$ \\
\hline Más de 5 años & 0,0147 & 0,0097 & 0,0197 & $16-18$ \\
\hline Escenario efectos mantenidos & 0,0126 & 0,0074 & 0,0195 & $16-18$ \\
\hline $\begin{array}{l}\text { Probabilidad anual de recaída a distancia después de recaída } \\
\text { local-regional }{ }^{*}\end{array}$ & 0,2 & 0,1847 & 0,2153 & 31 \\
\hline Probabilidad anual de embolismo pulmonar con tamoxifeno & 0,0098 & 0,0057 & 0,0139 & $16-17,19-21$ \\
\hline Probabilidad anual de cáncer de endometrio con tamoxifeno & 0,0017 & 0,0001 & 0,0035 & $16,17,19$ \\
\hline Probabilidad anual de enfermedad cerebrovascular con tamoxifeno & 0,0060 & 0,0028 & 0,0092 & $16,17,20$ \\
\hline Probabilidad anual de infarto de miocardio con tamoxifeno & 0,0050 & 0,0021 & 0,0080 & $16,17,19-21$ \\
\hline Probabilidad anual de fracturas con tamoxifeno** & 0,0115 & 0,0070 & 0,0159 & $16,17,19-21$ \\
\hline Probabilidad anual de embolismo pulmonar con anastrazol & 0,0056 & 0,0025 & 0,0087 & $16,17,19-21$ \\
\hline Probabilidad anual de cáncer de endometrio con anastrazol & 0,0003 & 0,0001 & 0,0010 & $16,17,19$ \\
\hline Probabilidad anual de enfermedad cerebrovascular con anastrazol & 0,0036 & 0,0011 & 0,0061 & $16,17,20$ \\
\hline Probabilidad anual de infarto de miocardio con anastrazol & 0,0076 & 0,0040 & 0,0112 & $16,17,19-21$ \\
\hline Probabilidad anual de fracturas con anastrazol** & 0,0186 & 0,0130 & 0,0242 & $16,17,19-21$ \\
\hline Probabilidad de morir por embolismo pulmonar & 0,15 & 0,07 & 0,18 & $22-23$ \\
\hline Probabilidad de morir por cáncer de endometrio & 0,20 & 0,16 & 0,25 & 25 \\
\hline Probabilidad de morir por infarto de miocardio & 0,10 & 0,06 & 0,28 & 26,27 \\
\hline Probabilidad de morir por enfermedad cerebrovascular & 0,14 & 0,13 & 0,17 & 28,29 \\
\hline Probabilidad de morir por fractura de cadera & 0,09 & 0,04 & 0,14 & $30-32$ \\
\hline Probabilidad anual de morir por enfermedad metastásica & 0,40 & 0,11 & 0,59 & $16,17,20$ \\
\hline \multicolumn{5}{|l|}{ Probabilidad de morir por otras causas } \\
\hline 50 años & 0,0034 & 0,0027 & 0,0041 & DANE 2005 \\
\hline 65 años & 0,0129 & 0,0103 & 0,0155 & DANE 2005 \\
\hline 80 o más años & 0,0915 & 0,0732 & 0,1098 & \\
\hline
\end{tabular}

* Las probabilidades de recaída después de cáncer del lado contrario se asumieron iguales a las del primer tumor.

** Se asumió que el $18 \%$ de las fracturas fueron de cadera.

modelo se trabajó sin tasa de descuento y con tasa de descuento del 3\% anual aplicada a efectos y costos, y sólo a costos. Los resultados se expresan para los tres casos.

El modelo se trabajó en el software Tree Age y se corrió a 30 años con los siguientes supuestos: las mujeres salen del modelo cuando sucede la primera recaída, cuando mueren por otras causas o cuando completan 30 años de seguimiento: lo que ocurra primero.

Se inicia con una cohorte de mujeres posmenopáusicas (50 años) con cáncer de mama operable con receptor hormonal positivo, que hayan completado el tratamiento quirúrgico y de 


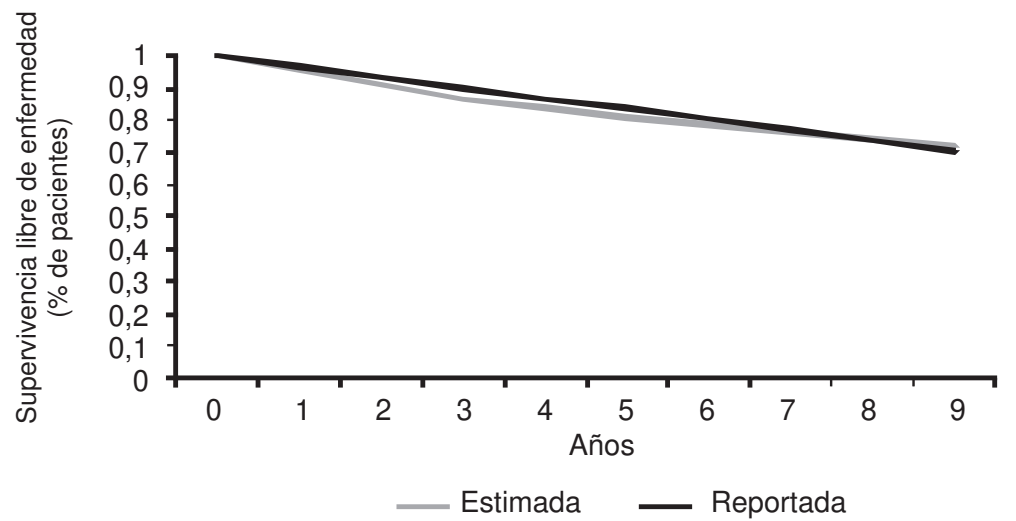

Figura 2. Funciones de supervivencia reportadas y estimadas.

Cuadro 2. Costo anual del tratamiento.

\begin{tabular}{|c|c|c|c|}
\hline & \multirow{2}{*}{$\begin{array}{l}\text { Costo base } \\
\text { (Col\$ de 2007) }\end{array}$} & \multicolumn{2}{|c|}{ Rango*** } \\
\hline & & Mínimo & Máximo \\
\hline Anastrazol* & $3{ }^{\prime} 285.000$ & 3'285.000 & 6'570.000 \\
\hline Tamoxifeno** & 109.500 & 109.500 & 547.500 \\
\hline Recaída local/regional & $44^{\prime} 377.580$ & $10 ’ 442.245$ & $239 ' 686.485$ \\
\hline Recaída a distancia & $28^{\prime} 706.580$ & $11^{\prime} 511.680$ & $220 ' 137.620$ \\
\hline Cáncer del lado contrario & $19 ' 837.071$ & $14 ' 257.212$ & $266^{\prime} 536.913$ \\
\hline Tromboembolia & 3'239.106 & $2^{\prime} 018.069$ & $19^{\prime} 847.881$ \\
\hline Accidente cerebrovascular & 4'797.640 & $2 ' 002.718$ & $66 ' 246.071$ \\
\hline Infarto de miocardio & $1^{\prime} 543.386$ & 234.400 & $133^{\prime} 831.933$ \\
\hline Fractura de cadera & $7 ’ 309.647$ & $5 ’ 020.039$ & $85 ' 124.595$ \\
\hline Fractura de muñeca & 705.647 & 200.550 & 3'305.800 \\
\hline Cáncer de endometrio & 2'565.180 & $2,074.025$ & 8'306.262 \\
\hline
\end{tabular}

*Para el análisis se tomaron precios por tableta de Col\$ 9.000 a Col\$18.000.

** Para el análisis se tomaron precios por tableta de Col\$300 a Col\$1.500.

quimioterapia primario. Después de cinco años de tratamiento adyuvante con hormonoterapia sin presentar recaída de la enfermedad, la efectividad se asumió igual para los dos tratamientos. Después de una recaída local o regional, estas pacientes no pueden experimentar una nueva recaída local o regional; sólo pueden experimentar una recaída a distancia. Se asumió que las muertes por cáncer de mama sólo ocurren después de una recaída a distancia. Las mujeres tratadas con tamoxifeno que presentan efectos adversos (embolismo pulmonar o cáncer de endometrio) se cambian a anastrazol, y las mujeres tratadas con anastrazol que presentan evento adverso (fracturas o infarto de miocardio) se cambian a tamoxifeno. Después de terminado el tratamiento no hay diferencias en las probabilidades de efectos adversos. El modelo supone total cumplimiento del tratamiento.

Con el objetivo de explorar el impacto sobre los resultados de costo-efectividad, se hicieron análisis de sensibilidad de una vía de las variables de costo, la efectividad y los supuestos. Se construyó un escenario conservador, al suponer que la efectividad (riesgo de recaída) es igual para ambos grupos después de terminar el periodo de tratamiento. Otro escenario trabajado fue el de efectos mantenidos, en el cual se asumió que la disminución en el riesgo de recaída con el anastrazol se mantiene después de terminar los cinco años de tratamiento. Este escenario se realizó teniendo en cuenta el último informe del estudio ATAC (21), el cual mostró que la efectividad del tratamiento con anastrazol se mantiene después de terminado el tratamiento.

Además, se realizaron análisis probabilísticos de sensibilidad con sus correspondientes regiones de confianza y curvas de aceptabilidad.

\section{Resultados}

Comparado con el tamoxifeno, el anastrazol genera un tiempo adicional libre de enfermedad de 0,49 años. El costo adicional por año de vida ganado 
libre de enfermedad con el anastrazol, utilizando los costos de tabletas de la cota inferior y sin descuento, es de Col\$27’210.604 por año de vida ganado libre de enfermedad. Con descuento del $3 \%$, la razón de costo-efectividad incremental es de Col\$37’071.337 por año de vida ganado libre de enfermedad (cuadro 3). Al aplicar el descuento del $3 \%$ sólo a los costos, las razones de costoefectividad incremental son de Col\$24'852.265 por año de vida ganado libre de enfermedad. Los resultados no fueron sensibles a los costos de recaídas y efectos adversos (figuras 3,4 y 5). En la figura 3, las barras muestran el rango de las razones de costo-efectividad incremental al modificar las variables dentro de los rangos definidos para los análisis de sensibilidad. La razón de costo-efectividad incremental es sensible al precio del anastrazol; esta intervención empieza a ser costo-efectiva a precios menores de Col\$2.309 por tableta. En el cuadro 4, se puede observar la importancia del peso de los medicamentos y los efectos adversos en el total de costos.
En el caso de efectos mantenidos cuyos resultados aparecen en el cuadro 5, se observa que las razones de costo-efectividad incremental con y sin descuento son de Col\$23'617.400 por año de vida ganado libre de enfermedad y Col\$16'140.282 por año de vida ganado libre de enfermedad, respectivamente.

Al hacer el análisis de sensibilidad probabilístico, la figura 4 muestra que la densidad de puntos bajo la diagonal definida por el umbral de disponibilidad a pagar (producto interno bruto per cápita de Col\$ 7'521.363), representa la frecuencia en que sería costo-efectivo el anastrazol comparado con el tamoxifeno, lo cual sucede en $4,15 \%$ de los casos.

Por su parte, la figura 5 muestra que el tamoxifeno tiene una mayor probabilidad de ser costo-efectivo comparado con el anastrazol, ante cualquier umbral.

El análisis de costo-efectividad muestra que, para Colombia, pasar de tamoxifeno a anastrazol para mujeres posmenopáusicas con cáncer de mama temprano receptor hormonal positivo no es costo-

Cuadro 3. Costo-efectividad de anastrazol versus tamoxifeno.

\begin{tabular}{|c|c|c|c|c|c|c|}
\hline & $\begin{array}{l}\text { Costo } \\
(\$)\end{array}$ & $\begin{array}{c}\text { Costo } \\
\text { incremental }\end{array}$ & $\begin{array}{l}\text { Efectividad } \\
\text { (AVGLE) }\end{array}$ & $\begin{array}{c}\text { Efectividad } \\
\text { incremental } \\
\text { (AVGLE) }\end{array}$ & $\begin{array}{l}\text { Razón C/E } \\
\text { (\$/AVGLE) }\end{array}$ & $\begin{array}{c}\text { Razón C/E } \\
\text { incremental } \\
\text { (\$/AVGLE) }\end{array}$ \\
\hline \multicolumn{7}{|l|}{ Sin descuento } \\
\hline Tamoxifeno 5 años & $16 ’ 586.126$ & & 17,659 & & 939.245 & \\
\hline Anastrazol 5 años & 29'919.322 & 13'.333.196 & 18,149 & 0,49 & $1^{\prime} 648.538$ & $27 ’ 210.604$ \\
\hline \multicolumn{7}{|l|}{ Con descuento } \\
\hline Tamoxifeno 5 años & $12 ’ 716.452$ & & 12,736 & & 998,465 & \\
\hline Anastrazol 5 años & $25 ' 246.564$ & $12 ’ 530.112$ & 13,074 & 0,338 & 1'931.051 & $37^{\prime} 071.337$ \\
\hline
\end{tabular}

AVGLE: años de vida ganados libres de enfermedad

C/E: costo-efectividad



Figura 3. Análisis de sensibilidad de una vía. 


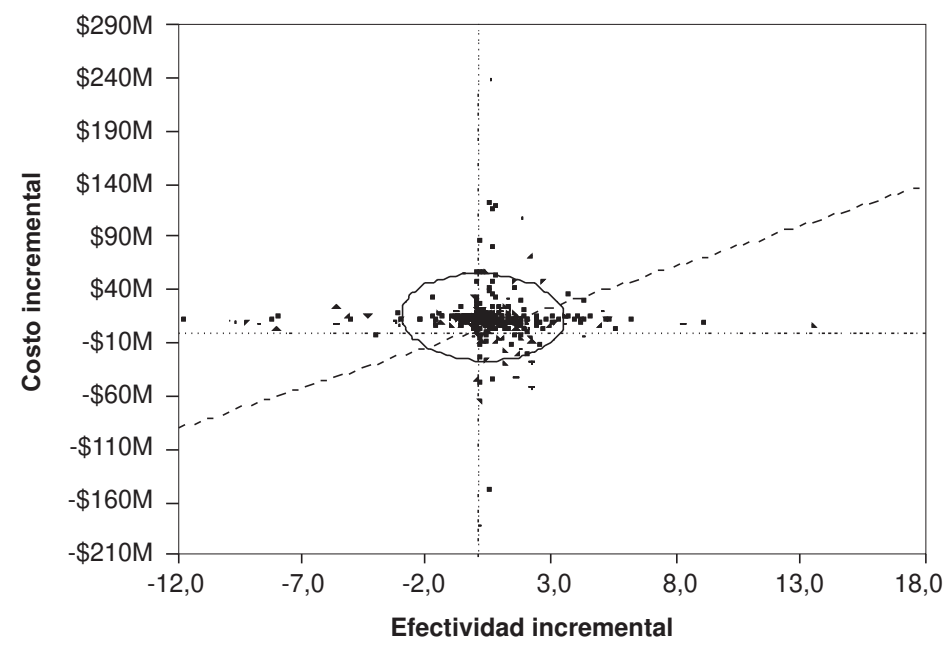

Figura 4. Región de confianza sobre el plano de efectividad anastrazol Vs. tamoxifeno

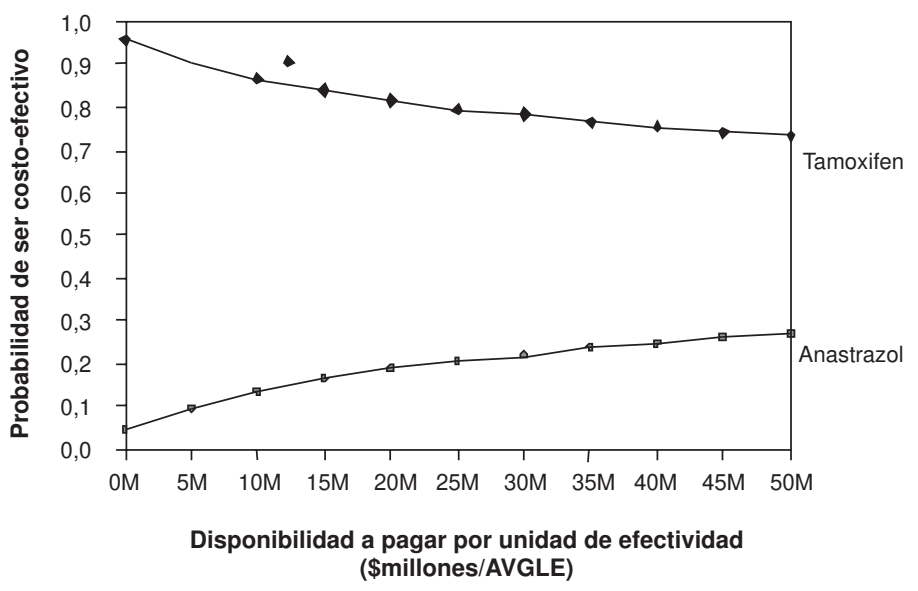

Figura 5. Curva de aceptabilidad

Cuadro 4. Participación de los costos para cada alternativa.

\begin{tabular}{lcccc}
\hline & Medicamento & Recaídas & Efectos secundarios & Total \\
\hline Anastrazol & $13^{\prime} 780.335$ & $10^{\prime} 763.724$ & 702.505 & $25^{\prime} 246.564$ \\
Tamoxifeno & 721.568 & $11^{\prime} 219.478$ & 775.406 & $12^{\prime} 716.452$ \\
\hline
\end{tabular}

efectivo, al tener el umbral establecido del producto interno bruto per cápita. Pasar de tamoxifeno a anastrazol como terapia inicial cuesta más de Col\$ 7’521.363 (producto interno bruto per cápita de Colombia en 2007) y, en consecuencia, no es costoefectivo para Colombia hacer esta transición.

En ese sentido, para el sistema de salud de Colombia, la alternativa costo-efectiva a los precios actuales es continuar con tamoxifeno como terapia inicial. Estos resultados son sólidos ante cambios en el costo de los efectos adversos. El anastrazol sólo sería recomendable a costos inferiores de Col\$2.309 por tableta.

\section{Discusión}

En mujeres posmenopáusicas con cáncer temprano de mama y receptor hormonal positivo, la terapia endocrina con tamoxifeno reduce la recurrencia y mortalidad por esta enfermedad (1). Si bien 
Cuadro 5. Costo-efectividad anastrazol versus tamoxifeno.

\begin{tabular}{|c|c|c|c|c|c|c|}
\hline Costo & $\begin{array}{l}\text { Costo } \\
(\$)\end{array}$ & $\begin{array}{c}\text { Costo } \\
\text { incremental }\end{array}$ & $\begin{array}{c}\text { Efectividad } \\
\text { (AVGLE) }\end{array}$ & $\begin{array}{c}\text { Efectividad } \\
\text { incremental } \\
\text { (AVGLE) }\end{array}$ & $\begin{array}{l}\text { Razón C/E } \\
\text { (\$/AVGLE) }\end{array}$ & $\begin{array}{c}\text { Razón C/E } \\
\text { incremental } \\
\text { (AVGLE) }\end{array}$ \\
\hline \multicolumn{7}{|l|}{ Sin descuento } \\
\hline Tamoxifeno 5 años & $16 ’ 348.037$ & & 17,739 & & 921.587 & \\
\hline Anastrazol 5 años & $29 ' 131.140$ & 12783.103 & 18,531 & 0,792 & 1'572.022 & $16^{\prime} 140.282$ \\
\hline \multicolumn{7}{|l|}{ Con descuento } \\
\hline Tamoxifeno 5 años & $12 ’ 572.171$ & & 12,779 & & 983.815 & \\
\hline Anastrazol 5 años & $24 ’ 687.897$ & $12 ' 115.726$ & 13,292 & 0,513 & 1'857.350 & $23^{\prime} 617.400$ \\
\hline
\end{tabular}

AVG: años de vida ganados

C/E: costo-efectividad

el anastrazol mejora la supervivencia libre de enfermedad, al ser comparada con el tamoxifeno (37) la diferencia de precios entre el tamoxifeno y el anastrazol en Colombia actúa a favor de la terapia convencional. No sucede lo mismo en otros países con costos de tecnologías y niveles de ingreso diferentes.

Los estudios sobre evaluación económica de la hormonoterapia son, en su mayoría, realizados para países de renta alta. Los umbrales de aceptación por año de vida ganado y ajustados por calidad para Estados Unidos y Canadá, llegan a los US\$100.000, en el Reino Unido, a las $£ 30.000$, en Suecia, a 500.000 coronas, en Holanda, a $20.000 €$ y en Bélgica, hasta $50.000 €(38-40)$. En consecuencia, la transición de tamoxifeno a anastrazol en esos países ha resultado aceptable dentro de esos parámetros, ya que los indicadores de costo-efectividad incremental calculados allí han sido inferiores a esos umbrales. Por ejemplo, el costo incremental por año de vida ganado con anastrazol se ha estimado en $£ 15.045$ y en US\$ 23.541-US\$ 75.000; mientras el costo incremental por año de vida ganado con calidad fue de $3.958 €$ y US\$ $20.246(11-13,40)$. No obstante, umbrales tan altos no pueden ser utilizados para tomar decisiones en países con condiciones económicas distintas. Aunque no existe consenso sobre cómo definir un umbral a partir del cual una nueva tecnología se considere costo-efectiva, la Organización Mundial de la Salud recomienda el producto interno bruto del país como una referencia para dicho umbral (35).

El estudio realizado presenta una limitación relacionada con la efectividad de la intervención, ya que los estudios publicados llevan periodos cortos de seguimiento y los resultados fueron extrapolados a horizontes de tiempo más largos. Sin embargo, en los análisis de sensibilidad en donde se mantiene la efectividad de la intervención, los resultados no cambian.
Si bien el anastrazol no es costo-efectivo a los precios incluidos en el modelo, el análisis de sensibilidad mostró que empezaría a ser costoefectivo a precios sustancialmente más bajos que los actuales (Col\$2.309 por tableta). El precio comercial de la tableta es actualmente cercano a los Col\$ 18.000. Este resultado merece ser tenido en cuenta por los tomadores de decisiones en el sector, tanto públicos como privados, y las empresas farmacéuticas en los procesos de negociación.

Este estudio se realizó para la población general. No obstante, es recomendable realizar el análisis para grupos de riesgo específicos, para los cuales se ha encontrado mayor eficacia de los inhibidores.

\section{Conflicto de intereses}

Los autores no tienen conflicto de intereses.

\section{Financiación}

Este trabajo se realizó con recursos del Instituto Nacional de Cancerología como parte de la Guía de Práctica Clínica explícita basada en la evidencia de hormonoterapia adyuvante en cáncer de mama, que se encuentra desarrollando dicha institución.

\section{Referencias}

1. Clarke M, Collins R, Darby S, Davies C, Evans V, Godwin $\mathbf{J}$, et al. Effects of chemotherapy and hormonal therapy for early breast cancer on recurrence and 15-year survival: an overview of the randomized trials (structured abstract). Lancet. 2005;365:1687-717.

2. Grana G. Adjuvant aromatase inhibitor therapy for early breast cancer: A review of the most recent data. J Surg Oncol. 2006;93:585-92.

3. Jones KL, Buzdar AU. A review of adjuvant hormonal therapy in breast cancer. Endocr Relat Cancer. 2004;11:391-406.

4. Bria E, Ciccarese M, Giannarelli D, Cuppone F, Nisticò C, Nuzzo C, et al. Early switch with aromatase inhibitors as adjuvant hormonal therapy for postmenopausal breast cancer: pooled-analysis of 8794 patients. Cancer Treat Rev. 2006;32:325-32. 
5. Cuppone F, Bria E, Verma S, Pritchard K, Gandhi S, Carlini P, et al. Do adjuvant aromatase inhibitors increase the cardiovascular risk in postmenopausal women with early breast cancer? Meta-analysis of randomized trials. Cancer. 2008;112:260-7.

6. Michaud LB. Adjuvant use of aromatase inhibitors in postmenopausal women with breast cancer. Am J Health Syst Pharm. 2005;62:266-73.

7. Mouridsen HT, Robert NJ. Benefit with aromatase inhibitors in the adjuvant setting for postmenopausal women with breast cancer. MedGenMed. 2005;7:20.

8. Locker G, Mansel R, Cella D, Dobrez D, Sorensen S, Gandhi S. Cost-effectiveness analysis of anastrozole versus tamoxifen as primary adjuvant therapy for postmenopausal women with early breast cancer: a US healthcare system perspective. The 5-year completed treatment analysis of the ATAC (Arimidex, tamoxifen alone or in combination) trial. Breast Cancer Res Treat. 2007;106:229-38.

9. Rocchi A, Verma S. Anastrozole is cost-effective Vs. tamoxifen as initial adyuvant therapy in early breast cancer: Canadian perspectives on the ATAC completed-treatment analysis. Support Care Cancer. 2006;14:917-27.

10. Moeremans K, Annemans L. Cost-effectiveness of anastrozole compared to tamoxifen in hormone receptorpositive early breast cancer. Analysis based on de ATAC trial. Int J Gynecol Cancer. 2006;16:576-8.

11. Kilian R, Porzsolt F. When to recommend and to pay for first-line adjuvant breast cancer treatment? A structured review of the literature. Breast. 2005;14:636-42.

12. Karnon J. Aromatase inhibitors in breast cancer. A review of cost considerations and cost effectiveness. Pharmacoeconomics. 2006;24:215-32.

13. Mansel R, Locker G, Fallowfield L, Benedict A, Jones D, On Behalf of the ATAC Trialists' Group. Costeffectiveness analysis of anastrozole Vs. tamoxifen in adjuvant therapy for early stage breast cancer in the United Kingdom: the 5-year completed treatment analysis of the ATAC (Arimidex, tamoxifen alone or in combination) trial. $\mathrm{Br}$ J Cancer. 2007;97:152-61.

14. Higa G. New generation aromatase inhibitors in breast cancer. Weighing out potential costs and beneficts. Pharmacoeconomics. 2000;2:121-32.

15. Wait S. Economic evaluation of endocrine therapy in the treatment of breast cancer. Anticancer Drugs. 1998;9:849-57.

16. Baum M, Budzar AU, Cuzick J, Forbes J, Houghton JH, Klijn JG, et al. Anastrozole alone or in combination with tamoxifen versus tamoxifen alone for adjuvant treatment of postmenopausal women with early breast cancer: first results of the ATAC randomized trial. Lancet. 2002;359:2131-9.

17. Howell A, Cuzick J, Baum M, Buzdar A, Dowsett M, Forbes JF, et al. ATAC Trialists' Group. Results of the ATAC (Arimidex, tamoxifen, alone or in combination) trial after completion of 5 years' adjuvant treatment for breast cancer. Lancet. 2005;365:60-2.

18. Goss PE, Ingle JN, Martino S, Robert N, Muss H, Piccart M, et al. Randomized trial of letrozole following tamoxifen as extended adjuvant therapy in receptor-positive breast cancer: updated findings from NCIC CTG MA.17. J Natl Cancer Inst. 2005;97:1262-71.
19. Jakesz R, Jonat W, Gnant M, Mittlboeck M, Greil R, Tausch C, et al. Switching of postmenopausal women with endocrine-responsive early breast cancer to anastrozole after 2 years' adjuvant tamoxifen: combined results of ABCSG trial 8 and ARNO 95 trial. Lancet. 2005;366:455-62.

20. Coates AS, Keshaviah A, Thurlimann B, Mouridsen $\mathrm{H}$, Mauriac L, Forbes J, et al. Five years of letrozole compared with tamoxifen as initial adjuvant therapy for postmenopausal women with endocrine-responsive early breast cancer: update of study BIG 1-98. J Clin Oncol. 2007;25:486-92.

21. Coombes RC, Kilburn LS, Snowdon CF, Paridaens R, Coleman RE, Jones SE, et al. Survival and safety of exemestane versus tamoxifen after 2-3 years' tamoxifen treatment (Intergroup Exemestane Study): a randomized controlled trial. Lancet. 2007;369:559-70.

22. British Thoracic Society Standards of Care Committee Pulmonary Embolism Guideline Development Group. British Thoracic Society guidelines for the management of suspected acute pulmonary embolism. Thorax. 2003;58:470-83.

23. Deitcher SR, Gomes MP. The risk of venous thromboembolic disease associated with adjuvant hormone therapy for breast carcinoma: a systematic review. Cancer. 2004;101:439-49.

24. Warwick D, Williams MH, Bannister GC. Death and thromboembolic disease after total hip replacement. A series of 1,162 cases with no routine chemical prophylaxis. J Bone Joint Surg Br. 1995;77:6-10.

25. MacDonald ND, Salvesen HB, Ryan A, Iversen OE, Akslen LA, Jacobs IJ. Frequency and prognostic impact of microsatellite instability in a large population-based study of endometrial carcinomas. Cancer Res. 2000;60:1750-2.

26. Rasmussen JN, Rasmussen S, Gislason GH, Buch P, Abildstrom SZ, Køber L, et al. Mortality after acute myocardial infarction according to income and education. J Epidemiol Community Health. 2006;60:351-6.

27. Kinoshita N, Imai K, Kinjo K, Naka M. Longitudinal study of acute myocardial infarction in the southeast Osaka district from 1988 to 2002. Circ J. 2005;69:1170-5.

28. Karagiannis A, Mikhailidis DP, Tziomalos K, Sileli M, Savvatianos S, Kakafika A, et al. Serum uric acid as an independent predictor of early death after acute stroke. Circ J. 2007;71:1120-7.

29. Kleindorfer D, Broderick J, Khoury J, Flaherty M, Woo D, Alwell $\mathrm{K}$, et al. The unchanging incidence and case-fatality of stroke in the 1990s: a population-based study. Stroke. 2006;37:2473-8.

30. Olmos JM, Martínez J, García J, Matorras P, Moreno JJ, González-Macías J. Incidencia de fractura de cadera en Cantabria. Med Clin (Barc). 1992;99:729-31.

31. Tarasevicius $\mathbf{S}$, Jermolajevas $\mathbf{V}$, Tarasevicius $\mathbf{R}$, Zegunis V, Smailys A, Kalesinskas RJ. Total hip replacement for the treatment of femoral neck fractures. Long-term results. Medicina (Kaunas). 2005;41:465-9.

32. Cornwall R, Gilbert MS, Koval KJ, Strauss E, Siu AL. Functional outcomes and mortality vary among different types of hip fractures: a function of patient characteristics. Clin Orthop Relat Res. 2004;425:64-71.

33. Schmoor C, Sauerbrei W, Bastert G, Schumacher M, for the German Breast Cancer Study Group. Role of Isolated 
locoregional recurrence of breast cáncer: results of four prospective studies. J Clin Oncol. 2000;18:1696-708.

34. Miller D, Homan SM. Determining transition probabilities: confusion and suggestions. Med Decis Making. 1994;14:52-8.

35. World Health Organization. Macroeconomics and health: investing in health for economic development: report of the Commission on Macroeconomics and Health. Geneva: World Health Organization; 2001.

36. Banco de la República. Estadísticas. Fecha de consultado: 15 de septiembre de 2008). Disponible en www.banrep.gov. co/estad/dsbbs/srea1_008.xls.

37. Forbes JF, Cuzick J, Buzdar A, Howell A, Tobias JS, Baum M. Effect of anastrozole and tamoxifen as adjuvant treatment for early-stage breast cancer: 100-month analysis of the ATAC trial. Lancet Oncol. 2008;9:45-53.

38. Jönson B. Changing health environment: The challenge to demonstrate cost-effectiveness of new compounds. Pharmacoeconomics. 2004;22:5-10.

39. Earle CC, Chapman RH, Baker CS, Bell C, Sandberg E, Neuman P. Systematic overview of cost-utility assessments in oncology. J Clin Oncol. 2000;18:3302-17.

40. Hillner BE. Benefit and projected cost-effectiveness of anatrozole versus tamoxifen as initial adjuvant therapy for patients with early stage estrogen receptor-positive breast cancer. Cancer. 2004:101:1311-22. 